\title{
Successful treatment of inadvertent subclavian artery cannulation by arterial closure device
}

\author{
Szu-Ling Chang, MD • Chih-Hung Lai, MD • \\ Hui-Chih Lai, MD, PhD • Chih-Jen Hung, MD • \\ Wen-Lieng Lee, MD, PhD
}

Received: 21 December 2016/Revised: 25 December 2016/ Accepted: 23 January 2017/Published online: 31 January 2017

(C) Canadian Anesthesiologists' Society 2017

\section{To the Editor,}

Central venous catheterization (CVC), a common procedure in perioperative and critical care settings, is associated with a mechanical complication rate of up to $34 \%$, including an arterial puncture incidence of $5 \%{ }^{1}$ Inadvertent cannulation of the subclavian artery is particularly challenging to address because its deep location and overlying structures make it difficult to address with compression or, if needed, a surgical approach. Immediate removal and compression after large-bore catheter insertion can lead to serious complications, including stroke and death. ${ }^{2}$ A catheterbased approach widely used to address femoral arterial puncture may offer a safe alternative for treating subclavian artery injury. ${ }^{3}$ We present a case of inadvertent subclavian artery cannulation managed with a percutaneous arterial closure device.

A 54-year-old male patient presented for trans-oral robotic operative management (with neck dissection) of a left-side stage III hypopharyngeal cancer. After anesthesia induction and endotracheal intubation, right subclavian vein access was obtained (using a surface landmark approach) with an $18 \mathrm{G}$ needle. After non-pulsatile

S.-L. Chang, MD - H.-C. Lai, MD, PhD - C.-J. Hung, MD Department of Anesthesiology, Taichung Veterans General Hospital, Taichung, Taiwan

S.-L. Chang, MD - C.-H. Lai, MD - H.-C. Lai, MD, PhD ·

W.-L. Lee, MD, PhD

School of Medicine, National Yang-Ming University, Taipei,

Taiwan

C.-H. Lai, MD - W.-L. Lee, MD, PhD ( $ه)$

Cardiovascular Center, Taichung Veterans General Hospital, Taichung, Taiwan

e-mail: wenliengleevghtc@gmail.com backflow appeared to be venous, an 8-Fr CVC was placed over a guidewire. Pulsatile flow was then observed from the CVC side port, however, indicating inadvertent subclavian artery cannulation. The catheter was left in place, and a cardiovascular surgeon was consulted. Because the surgical approach for repair was anticipated to be difficult, percutaneous treatment was chosen.

Angiography, with contrast injection via the CVC, confirmed the puncture site (Figure A). The cardiologist then performed a percutaneous endovascular repair using an arterial closure device (Angio-seal VIP 6-Fr system; St. Jude Medical; Minnetonka, MN, USA) that was inserted via the puncture site and sealed the wound successfully (Figure B). After the intervention, follow-up radiography of the chest showed no hemothorax or pneumothorax. The next day, ultrasonography of the site revealed a patent artery with good flow (Figure C). One month later, computed tomography also showed normal patency of the right subclavian artery and absorption of the previously placed closure device.

Inadvertent arterial injury following CVC insertion with a large-bore catheter $(\geq 7 \mathrm{Fr}$ ) has led to serious complications, including hematoma, arteriovenous fistula, pseudoaneurysm, hemothorax, stroke, and even death. ${ }^{2}$ Despite the many methods that have been reported to prevent arterial placement of CVCs (i.e., ultrasound guidance, pressure waveform analysis, blood gas analysis, manometry), ${ }^{4}$ inadvertent arterial cannulation can still occur.

Once inadvertent arterial cannulation is recognized, management of arterial injuries depends on many factors, including patient stability, catheter diameter, and whether the catheter is still in place. Pull and compression methods have been reported to be associated with significantly more instances of morbidity and mortality than surgical or 

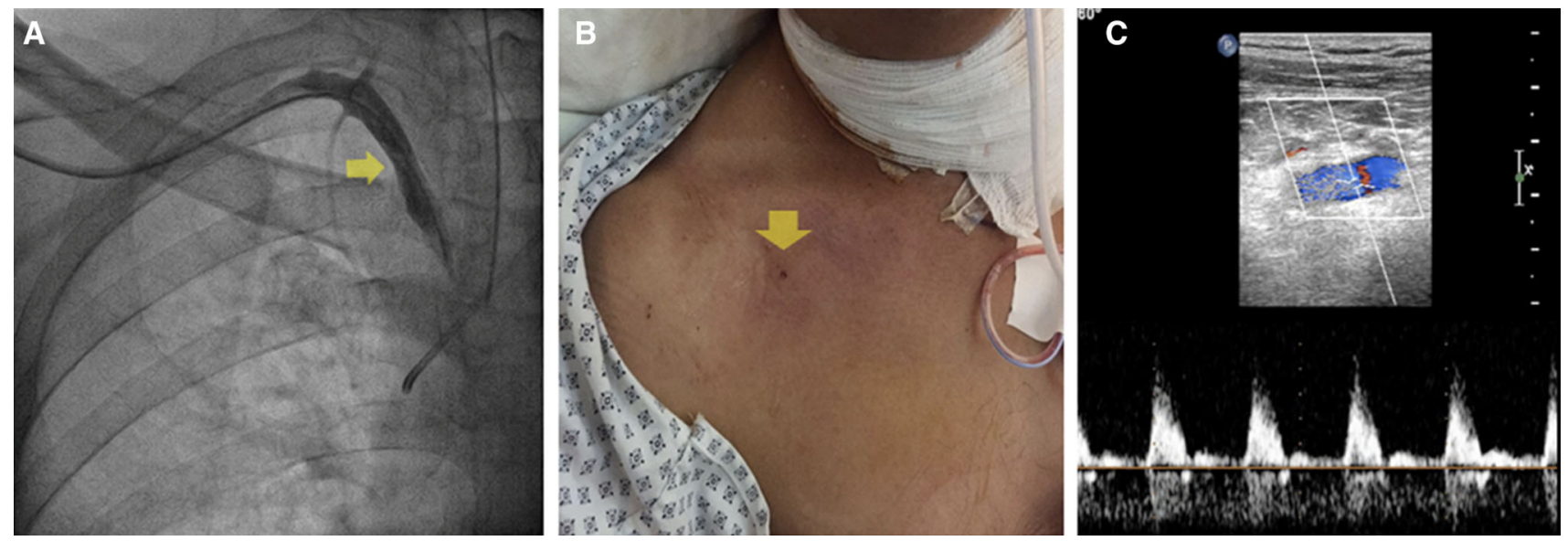

Figure (A) Angiography performed through a central venous catheter confirmed its inadvertent placement in the right subclavian artery (arrow). (B) The arterial wound was closed percutaneously

endovascular management $(46 \%$ vs $0 \%) .^{2}$ Thus, immediate removal of a large-bore catheter before vascular surgery consultation should be avoided.

Because of its deep location, an open surgical approach to the subclavian artery could be challenging and produce serious complications. Thus, a percutaneous endovascular approach to management of an injured subclavian artery is a potential alternative treatment, particularly in hemodynamically stable cases. ${ }^{3}$ In our case, we chose an arterial closure device (Angio-Seal), initially designed for femoral artery closure, to seal the puncture site. Hemostasis with these devices (recommended for vessels $>4 \mathrm{~mm}$ diameter) is achieved by means of a collagen sponge that is anchored to the arteriotomy site (see video at https://www. sjmglobal.com/en-int/professionals/resources-and-reimbur sement/video-and-media/vas-angio-seal-vip-demonstration). Compared with surgical repair, it is a simple, effective approach - although not free of complications, including embolization, occlusion, and laceration of the puncture site. ${ }^{5}$ Ultrasonography and clinical follow-up are recommended.

Conflicts of interest None declared. with an Angio-seal VIP 6-Fr system (St. Jude Medical; Minnetonka, MN, USA) without hematoma (arrow). (C) Follow-up ultrasonography showed good patency of the distal subclavian artery.

Editorial responsibility This submission was handled by Dr. Hilary P. Grocott, Editor-in-Chief, Canadian Journal of Anesthesia.

Funding None.

\section{References}

1. Eisen LA, Narasimhan M, Berger JS, Mayo PH, Rosen MJ, Schneider $R F$. Mechanical complications of central venous catheters. J Intensive Care Med 2006; 21: 40-6.

2. Guilbert MC, Elkouri S, Bracco D, et al. Arterial trauma during central venous catheter insertion: case series, review and proposed algorithm. J Vasc Surg 2008; 48: 918-25.

3. Carrick MM, Morrison CA, Pham HQ, et al. Modern management of traumatic subclavian artery injuries: a single institution's experience in the evolution of endovascular repair. Am J Surg 2010; 199: 28-34.

4. Gibson F, Bodenham A. Misplaced central venous catheters: applied anatomy and practical management. Br J Anaesth 2013; 110: 333-46.

5. Kalapatapu VR, Ali AT, Masroor F, Moursi MM, Eidt JF. Techniques for managing complications of arterial closure devices. Vasc Endovasc Surg 2006; 40: 399-408. 